\title{
Y-balance-test en jugadores de fútbol atendiendo al nivel de competición Y-balance-test in soccer players according to the competition level
}

*Moisés Falces Prieto, **Salvador Baena Morales, *Adrián Benítez Jiménez, *Rodrigo Revilla Gil, ***Alberto Muñoz Muñoz, ****Víctor Salinas Palacios, *****Rubén Betanzos López, ******Juan Jesús Adalid Leiva, ******* Francisco Tomás González Fernández

*Departamento Optimización del Rendimiento Físico Marcet, Barcelona (España), **Universidad Pontifica de Comillas (España), ***Football Club Sochaux-Montbéliard (Francia), ****Instituto de Salud y Deporte, Murcia (España), *****Extremadura CF (España), ****** Antequera CF (España), ******* Universidad de Granada (España)

Resumen. El presente estudio pretende mostrar de forma descriptiva los rangos de normalidad del Y-Balance-Test (YBT) para 173 jugadores de fútbol masculino, agrupados en 5 categorías (Cadetes: $\mathrm{n}=48$; edad=14.18 \pm 2.02 años; Juveniles: $\mathrm{n}=62$; edad=18.20 \pm 2.04 años; $3^{a}$ División: $\mathrm{n}=21$; edad $=25.42 \pm 4.52$ años; $2^{a}$ División $B: \mathrm{n}=19$; edad $=25.68 \pm 3.00$ años; $1^{a}$ División: $\mathrm{n}=23$; edad $=32.91$ \pm 3.04 años) y diferenciando la posición específica (portero, lateral, defensa central, centrocampista, extremo y delanteros). Los resultados del presente estudio se presentan en medias y desviaciones típicas, con el fin de poder mostrar el alcance normalizado (pierna dominante, no dominante) y las asimetrías entre extremidades (promedio $\pm \mathrm{DE}$; Total $\mathrm{d} » \pm 4 \mathrm{y} \%$ del total en $\mathrm{cm}$ ). Aunque es un estudio descriptivo, los datos que aquí se encuentran, parecen mostrar una mejora en los rangos del YBT a medida que aumentan la categoría para todas las medidas evaluadas. De forma complementaria, podemos sugerir que los delanteros de todas las categorías presentan déficit en la medida posteromedial (PM) y los jugadores de categoría cadete y juvenil, concretamente en la posición de centrocampistas, poseen grandes déficits en la totalidad de las medidas con respecto a los demás participantes del estudio. En conclusión, los resultados obtenidos nos permitirán obtener un amplio espectro de medidas en el YBT para diferentes categorías, que hasta donde alcanza nuestro conocimiento no existe en la literatura, para así ser más precisos en su evaluación. Además, nos ayudará a conocer con más precisión los rangos de normalidad de cada uno de los grupos, valorando en cada momento como la edad de estos influye en las asimetrías que podemos encontrar en nuestros futbolistas, permitiéndonos actuar en el deportista y establecer programas preventivos individualizados y preventivos más eficientes.

Palabras clave: Evaluación, Rango de movimiento, Profesional, Jugadores jóvenes, Control postural.

Abstract. The aim of this study is to show the ranges of normality for soccer players in different categories evaluated by Y-Balance-Test (YBT). A total of 173 soccer players were evaluated and grouped in 5 different categories (U16: $\mathrm{n}=48$; age $=14.18 \pm 2.02$ age; $U 19$ : $\mathrm{n}=$ 62; age = $18.20 \pm 2.04$ age; Third Division: $\mathrm{n}=21$; age $=25.42 \pm 4.52$ age; Second Division $B: \mathrm{n}=19$; age $=25.68 \pm 3.00$ age; First Division: $\mathrm{n}=23$; age $=32.91 \pm 3.04$ age) according to their specific positions (goalkeeper, full backs, central defenders, center midfielders, wingers, and center forwards). The results of this research consist in the means and standard deviation for the YBT, with the purpose of explaining the standardized reach (dominant and non-dominant legs) and asymmetries between limbs (average $\pm \mathrm{DE}$; Total d» \pm 4 and $\%$ of the total in $\mathrm{cm}$ ). While the nature of this study is purely observational, an increase in the scores for all the measurements can be noted from lower to higher categories. At all categories, center forwards present a deficit in the posteromedial movement (PM), whereas center midfielder from U16 and U19 is the position with the highest deficits. The knowledge of the YBT scores is essential to understand with greater precision how categories influence the asymmetries, and consequently to create more effective preventive programs.

Key words: Evaluation, Range of movement, Professional, Young players, Posture control.

\section{Introducción}

En la actualidad, los especialistas en ciencias del deporte han incrementado su interés en la valoración del control postural para la prevención de lesiones y recidivas de forma general, aunque principalmente en las extremidades inferiores (Gribble \& Hertel, 2003; Meardon, Klusendorf, \& Kernozek, 2016; Plisky, Rauh, Kaminski, \& Underwood, 2006). En este sentido, los anteriores estudios se han realizado de forma general para el seguimiento y evaluación del deportista, obteniendo por parte de los científicos deportivos información objetiva con la que realizar comparaciones en diferentes momentos de la temporada o en determinados momentos puntuales (Troule \& Casamichanana, 2016), como por ejemplo en el momento de return to play o vuelta a jugar después de una lesión (Gonell, Romero, \& Soler, 2015; Gribble \& Hertel, 2003; Hewit, Cronin, \& Hume, 2012; Plisky et al., 2006) y así, establecer parámetros de referencia para el ámbito científico. Concretamente, las asimetrías o desequilibrios

Fecha recepción: 25-02-19. Fecha de aceptación: 07-10-19 Moisés Falces Prieto mfalpri@gmail.com funcionales son uno de los focos más importantes en los que centran su atención los especialistas en ciencias del deporte, ya que, ha sido ampliamente demostrado que podrían afectar al rendimiento deportivo e incrementar la incidencia lesional (Troule \& Casamichanana, 2016). Por lo tanto, sería de gran relevancia estudiar las asimetrías funcionales debido a la valiosa información que nos proporciona de forma pronóstica y diagnóstica (Atkins, Bentley, Hurst, Sinclair, \& Hesketh, 2016).

La valoración clínica del equilibrio se ha basado a lo largo de décadas en pruebas de baja fiabilidad, susceptibles a la subjetividad del evaluador y, en la mayoría de los casos, poco eficientes como medida diagnóstica (Leirós-Rodríguez, Arce-Fariña, \& García-Soidán, 2015). Así, cuando hablamos más específicamente acerca de la estabilidad postural, o equilibrio, podríamos definirlo como la capacidad para mantener el centro de masa corporal dentro de la base de sustentación (Vanmeerhaeghe et al., 2009). Por otro lado, la orientación postural, hace referencia a la capacidad para mantener una correcta relación entre los propios segmentos del cuerpo y entre éstos y el entorno a la hora de realizar la tarea (Ageberg, Roberts, Holmström, \& Fridén, 2005). Dicho control postural 
se mantiene gracias a la integración dinámica de fuerzas internas y externas reguladas por estímulos visuales, vestibulares y somatosensoriales, es decir, gracias al control neuromuscular (Bressel, Yonker, Kras, \& Heath, 2007; Gribble, Tucker, \& White, 2007; Gribble, Hertel, \& Plisky, 2012; Mancera-Soto, Hernández-Álvarez, Hernández-Salinas, PrietoMondragon, \& Quiroga-Díaz, 2013; Lee, Lin, \& Huang, 2006).

En el caso del fútbol, numerosas investigaciones actuales ponen de manifiesto la importancia de la estabilidad postural en el desarrollo físico del jugador y en las tareas relacionadas con el propio juego (Keith, Condon, Phillips, McKeon, \& King, 2016; Mcguine, Greene, Best, \& Leverson, 2000), así como en la prevención y/o readaptación de lesiones (Sclafani \& Davis, 2016). Además, existen evidencias previas acerca de la importancia del balance postural, concretamente para mantener la estabilidad y el dominio de la posición del cuerpo durante el juego (Mancera-Soto et al., 2013).

De acuerdo con lo anteriormente mencionado, una herramienta que evalúa dicho control postural dinámico en futbolistas es el Y-Balance-Test (YBT) (Butler, Queen, Beckman, Kiesel \& Plisky, 2013). El YBT se ha mostrado como una herramienta válida y fiable para predecir lesiones futuras en las extremidades inferiores (Butler, Southers, Gorman, Kiesel \& Plisky, 2012; González et al., 2011: Gribble et al., 2012; Keith et al., 2016; Plisky et al., 2009; Plisky et al., 2006). Esta prueba es una variación del Star Excursion Balance Test (SEBT), la cual, reduce el test inicial (SEBT) de ocho direcciones a tres: anterior (ANT), postero-medial (PM) y Postero-lateral (PL) (Hertel, Braham, Hale, \& Olmsted-Kramer, 2006; Langarica-Rocafort, 2014; Robinson \& Gribble, 2008). Con respecto a nivel de confiabilidad en una escala de 0 a 3 , el YBT presenta un valor de 3 a nivel de protocolo, objetividad, fiabilidad y validez, por lo que, presenta una alta confiabilidad para medir la capacidad de equilibrio (Triana \& Espitia, 2019). En consecuencia, para valorar futbolistas sería un test de fácil aplicación, sencillo y muy útil que nos proporcionará medidas importantes para el objetivo de nuestro estudio.

El propósito del presente estudio pretende mostrar de forma descriptiva los rangos de normalidad del YBT para 173 jugadores de fútbol masculino, agrupados en 5 diferentes categorías. Para ello nos basamos en un test que ha sido ampliamente utilizado en futbolistas (Butler et al., 2013; Gonell et al., 2015). Hasta donde alcanza nuestro conocimiento, no conocemos ningún estudio descriptivo, ni longitudinal que establezca rangos de normalidad con el YBT en distintas categorías y puestos específicos. Tampoco conocemos ningún estudio con una muestra tan amplia de jugadores de fútbol. Es por ello, que destacamos la importancia de este, ya que, nos ayudará a determinar rangos de normalidad atendiendo a la categoría (Cadete, Juvenil, $3^{\mathrm{a}}$ División, $2^{\text {a }}$ división B y $1^{a}$ División) y puestos específicos (portero, lateral, defensa central, centrocampista, extremo y delanteros).

\section{Material y Métodos}

\section{Participantes}

173 jugadores de fútbol masculino, agrupados en 5 categorías (Cadetes: $n=48$; edad=14.18 \pm 2.02 años; altura=171.09 \pm 2.02 cm; Juveniles: $n=62$; edad= $18.20 \pm 2.04$ años; altu$\mathrm{ra}=172.37 \pm 8.26 \mathrm{~cm}$; $3^{\mathrm{a}}$ División: $\mathrm{n}=21$; edad $=25.42 \pm 4.52$ años; altura $=180.04 \pm 5.29 \mathrm{~cm}$; $2^{\text {a }}$ División B: $\mathrm{n}=19$; edad = $25.68 \pm 3.00$ años; altura $=180 \pm 5.93$ cm; $1^{\text {a }}$ División: $\mathrm{n}=23$; edad $=32.91 \pm 3.04$ años; altura $=180.13 \pm 5.35 \mathrm{~cm}$ ) con más de 5 años en la práctica deportiva federada. (Ver tabla 1). La muestra de categoría cadete y juvenil fue seleccionada de una academia de alto rendimiento en fútbol que competían en competición federada de ámbito autonómico. La muestra de jugadores de $3^{\mathrm{a}}$ División, $2^{\mathrm{a}}$ División $\mathrm{B}$ y $1^{\mathrm{a}}$ División, fueron jugadores que competían de forma federada bajo la jurisdicción de la Real Federación Española de Fútbol.

Los criterios de inclusión fueron: tener entre 14 y 19 años (para la categoría cadete y juvenil), no tener ninguna enfermedad que impidiese la práctica de actividad física, no tener antecedentes de trastornos neurológicos o físicos y dieron su consentimiento informado antes del inicio del experimento. Además de entregar a los sujetos el protocolo del procedimiento para la realización de los test físicos y una explicación personal, todos los jugadores, padres-madres y/o tutores, se les notificó el diseño de la investigación y sus requisitos, así como de los beneficios y riesgos potenciales antes de iniciar el estudio.

Los criterios de exclusión usados fueron que manifestaran antecedentes de lesión en tobillo o rodilla en los últimos tres meses (5 jugadores) y estar recibiendo atención fisioterapéutica por lesión (6 jugadores). El trabajo fue diseñado respetando las normas de la Declaración de Helsinki y aprobado por el comité ético de la Universidad Internacional de Valencia.

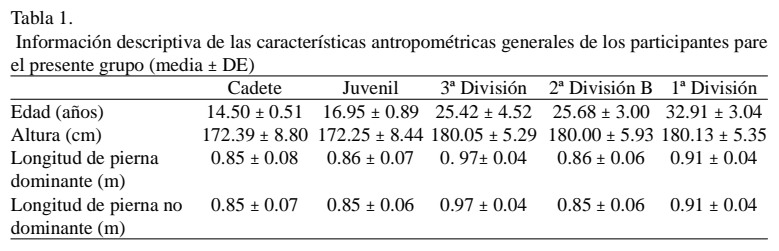

\section{Material}

El control de la altura de los jugadores fue controlado por un estadímetro de pared SECA ${ }^{\circledR}$ (modelo 206, Hamburgo, Alemania). Para la medición de la longitud de cada pierna se utilizó una cinta métrica clásica modelo MM0231, con una cara graduada en centímetros, un largo de 3 metros y ancho de 2 centímetros. Para la medición de la longitud de ambos miembros inferiores en cada sujeto, se llevó a cabo la medición desde la espina iliaca antero-superior a maléolo tibial (Gribble \& Hertel, 2003) con una cinta métrica clásica modelo MM0231, con una cara graduada en centímetros, un largo de 2 metros y ancho de 2 centímetros. Aunque esta medición no es antropométricamente correcta, se realizó de esta forma debido a que es así como se determina en la normalización del test y en otros trabajos referenciados (González et al., 2011). Los datos fueron recogidos en una ficha formato papel y posteriormente pasados a una hoja informatizada de Excel de Microsfot Office ${ }^{\circledR}$.

\section{Procedimiento}

En primer lugar, se determinó la pierna dominante preguntando a los participantes con que pierna golpean lo más fuerte posible y con mayor precisión a un balón. Para la 
realización de la prueba, el participante se descalzó para facilitar la colocación del pie y eliminar la variabilidad debida al uso del calzado. El YBT se llevó a cabo con 3 cintas métricas clásicas modelo MM0231, adheridas al suelo con un ángulo

Tabla 2.

Alcance normalizado entre pierna dominante y no dominante y asimetrías entre extremidades: Diferencia entre pierna dominante y no dominante. Categoría Cadete.

\begin{tabular}{|c|c|c|c|c|c|c|}
\hline \multirow{2}{*}{ Posición en el campo } & & \multicolumn{2}{|c|}{ Alcance normalizado } & \multicolumn{3}{|c|}{ Déficit Bilateral } \\
\hline & & $\begin{array}{c}\text { Pierna } \\
\text { Dominante }\end{array}$ & $\begin{array}{c}\text { Pierna } \\
\text { no dominante }\end{array}$ & $\begin{array}{l}\text { Prom } \\
\pm \mathrm{DE}\end{array}$ & $\begin{array}{c}\text { Total }= \pm \\
4 \mathrm{~cm}\end{array}$ & $\begin{array}{l}\text { \% del } \\
\text { Total }\end{array}$ \\
\hline & ANT & $68.42 \pm 7.71$ & $69.22 \pm 8.87$ & $0.04 \pm 0.01$ & 2 & 40.00 \\
\hline \multirow[t]{3}{*}{ Portero $(n=5)$} & PL & $104.70 \pm 11.33$ & $108.66 \pm 12.60$ & $0.11 \pm 0.05$ & 5 & 100 \\
\hline & PM & $99.72 \pm 8.95$ & $105.45 \pm 8.28$ & $0.52 \pm 0.04$ & 2 & 40.00 \\
\hline & ANT & $72.04 \pm 9.22$ & $71.88 \pm 8.25$ & $0.37 \pm 0.28$ & 2 & 28.57 \\
\hline \multirow[t]{3}{*}{ Lateral (n=7) } & PL & $110.55 \pm 16.87$ & $110.61 \pm 13.82$ & $0.62 \pm 0.26$ & 6 & 85.71 \\
\hline & PM & $104.50 \pm 9.42$ & $105.80 \pm 14.61$ & $0.64 \pm 0.45$ & 5 & 71.42 \\
\hline & ANT & $68.67 \pm 13.04$ & $67.52 \pm 11.34$ & $0.26 \pm 0.29$ & 3 & 50.00 \\
\hline \multirow[t]{3}{*}{ Defensa Central $(n=6)$} & PL & $109.13 \pm 12.91$ & $106.45 \pm 12.08$ & $0.36 \pm 0.04$ & 3 & 50.00 \\
\hline & PM & $102.72 \pm 10.36$ & $102.49 \pm 13.40$ & $0.33 \pm 0.25$ & 2 & 33.33 \\
\hline & ANT & $69.76 \pm 8.24$ & $69.74 \pm 8.55$ & $0.26 \pm 0.02$ & 4 & 22,22 \\
\hline \multirow[t]{3}{*}{ Centrocampista $(\mathrm{n}=18)$} & PL & $105.79 \pm 8.96$ & $107.95 \pm 8.70$ & $0.28 \pm 0.02$ & 6 & 33.33 \\
\hline & PM & $101.82 \pm 10.25$ & $99.97 \pm 13.48$ & $0.60 \pm 0.06$ & 9 & 50.00 \\
\hline & ANT & $79.85 \pm 12.54$ & $76.21 \pm 8.22$ & $0.04 \pm 0.06$ & 1 & 16.66 \\
\hline \multirow[t]{3}{*}{ Extremo $(\mathrm{n}=6)$} & PL & $102.50 \pm 10.70$ & $107.54 \pm 11.08$ & $0.08 \pm 0.04$ & 4 & 66.66 \\
\hline & PM & $104.38 \pm 9.06$ & $100.42 \pm 4.43$ & $0.05 \pm 0.05$ & 3 & 50.00 \\
\hline & ANT & $70.86 \pm 8.14$ & $71.51 \pm 8.62$ & $0.02 \pm 0.01$ & 1 & 22.22 \\
\hline \multirow[t]{2}{*}{ Delantero (n=6) } & PL & $106.19 \pm 6.31$ & $107.55 \pm 10.27$ & $0.04 \pm 0.04$ & 2 & 33.33 \\
\hline & PM & $107.49 \pm 11.56$ & $106.33 \pm 9.51$ & $0.06 \pm 0.04$ & 3 & 50.00 \\
\hline
\end{tabular}

Tabla 3.

Alcance normalizado entre pierna dominante y no dominante y asimetrías entre extremidades: Diferencia entre pierna dominante y no dominante. Categoría Juvenil.

\begin{tabular}{|c|c|c|c|c|c|c|}
\hline \multirow{2}{*}{\multicolumn{2}{|c|}{ Posición en el campo }} & \multicolumn{2}{|c|}{ Alcance normalizado } & \multicolumn{3}{|c|}{ Déficit bilateral } \\
\hline & & \multirow{3}{*}{$\begin{array}{c}\text { Pierna } \\
\text { Dominante } \\
71.18 \pm 7.56\end{array}$} & \multirow{3}{*}{$\begin{array}{c}\begin{array}{c}\text { Pierna } \\
\text { no dominante }\end{array} \\
70.65 \pm 9.84\end{array}$} & \multirow{3}{*}{$\begin{array}{c}\text { Prom } \\
\pm \mathrm{DE} \\
0.03 \pm 0.04\end{array}$} & \multirow{3}{*}{$\begin{array}{c}\text { Total }= \pm \\
4 \mathrm{~cm}\end{array}$} & \multirow{3}{*}{$\begin{array}{l}\begin{array}{c}\% \text { del } \\
\text { Total }\end{array} \\
50.00\end{array}$} \\
\hline & & & & & & \\
\hline \multirow{4}{*}{ Portero $(\mathrm{n}=8)$} & ANT & & & & & \\
\hline & PL & $110.41 \pm 10.31$ & $110.94 \pm 13.48$ & $0.04 \pm 0.03$ & 3 & 37.50 \\
\hline & PM & $98.86 \pm 16.26$ & $105.35 \pm 16.67$ & $0.04 \pm 0.03$ & 6 & 75.00 \\
\hline & ANT & $69.72 \pm 7.95$ & $71.21 \pm 6.37$ & $0.05 \pm 0.02$ & 5 & 50.00 \\
\hline \multirow[t]{3}{*}{ Lateral $(\mathrm{n}=10)$} & PL & $105.37 \pm 14.41$ & $107.57 \pm 9.26$ & $0.08 \pm 0.08$ & 7 & 70.00 \\
\hline & PM & $100.29 \pm 6.22$ & $99.38 \pm 10.59$ & $0.07 \pm 0.04$ & 8 & 80.00 \\
\hline & ANT & $69.77 \pm 3.85$ & $70.15 \pm 5.20$ & $0.02 \pm 0.01$ & 3 & 30.00 \\
\hline \multirow[t]{3}{*}{ Defensa Central $(\mathrm{n}=10)$} & & $105.43 \pm 17.10$ & $107.60 \pm 13.68$ & $0.03 \pm 0.02$ & 6 & 60.00 \\
\hline & PM & $101.61 \pm 14.66$ & $103.72 \pm 12.35$ & $0.06 \pm 0.06$ & 5 & 50.00 \\
\hline & ANT & $67.51 \pm 7.18$ & $66.52 \pm 9.26$ & $0.03 \pm 0.02$ & 8 & 40.00 \\
\hline \multirow[t]{3}{*}{ Centrocampista $(\mathrm{n}=20)$} & PL & $106.14 \pm 11.01$ & $108.16 \pm 12.40$ & $0.04 \pm 0.04$ & 9 & .33 \\
\hline & PM & $101.50 \pm 11.49$ & $102.56 \pm 12.54$ & $0.06 \pm 0.04$ & 13 & 50.00 \\
\hline & ANT & $71.10 \pm 5.97$ & $71.07 \pm 5.69$ & $0.03 \pm 0.02$ & 3 & 16.66 \\
\hline \multirow[t]{3}{*}{ Extremo $(\mathrm{n}=8)$} & PL & $108.30 \pm 9.76$ & $107.89 \pm 8.05$ & $0.04 \pm 0.04$ & 3 & 66.66 \\
\hline & PM & $102.91 \pm 12.56$ & $106.40 \pm 7.86$ & $0.06 \pm 0.06$ & 4 & 50.00 \\
\hline & ANT & $69.55 \pm 7.41$ & $71.17 \pm 8.56$ & $0.02 \pm 0.02$ & 1 & 22.22 \\
\hline \multirow[t]{2}{*}{ Delantero $(\mathrm{n}=6)$} & PL & $108.48 \pm 8.56$ & $108.25 \pm 10.77$ & $0.04 \pm 0.03$ & 3 & 33.33 \\
\hline & PM & $101.16 \pm 10.01$ & $98.50 \pm 10.57$ & $0.06 \pm 0.04$ & 4 & 50.00 \\
\hline \multicolumn{7}{|c|}{$\begin{array}{l}\text { Tabla } 4 . \\
\text { Alcance normalizado entre pierna dominante y no dominante y asimetrías entre extremidades: } \\
\text { Diferencia entre pierna dominante y no dominante. Tercera División. }\end{array}$} \\
\hline & & \multicolumn{2}{|c|}{ Alcance normalizado } & \multicolumn{3}{|c|}{ Déficit bilateral } \\
\hline \multirow{2}{*}{\multicolumn{2}{|c|}{ Posición en el campo }} & Pierna & Pierna & Prom & Total $= \pm$ & $\% \mathrm{del}$ \\
\hline & & Dominante & no dominante & $\pm \mathrm{DE}$ & $4 \mathrm{~cm}$ & Total \\
\hline & ANT & $103.98 \pm 1.22$ & $104.53 \pm 0.45$ & $0.00 \pm 0.00$ & 0 & 00.00 \\
\hline \multirow[t]{3}{*}{ Portero $(\mathrm{n}=3)$} & PL & $106.66 \pm 5.30$ & $106.71 \pm 5.38$ & $0.00 \pm 0.00$ & 0 & 00.00 \\
\hline & PM & $107.70 \pm 6.77$ & $107.85 \pm 9.73$ & $0.01 \pm 0.01$ & 0 & 00.00 \\
\hline & ANT & $105.01 \pm 9.29$ & $104.65 \pm 8.35$ & $0.02 \pm 0.02$ & 2 & 50.00 \\
\hline \multirow[t]{3}{*}{ Lateral $(\mathrm{n}=4)$} & PL & $123.18 \pm 4.15$ & $127.42 \pm 3.58$ & $0.04 \pm 0.04$ & 2 & 50.00 \\
\hline & PM & $121.09 \pm 3.84$ & $122.32 \pm 8.69$ & $0.04 \pm 0.02$ & 2 & 50.00 \\
\hline & ANT & $87.98 \pm 10.05$ & $93.01 \pm 8.57$ & $0.09 \pm 0.06$ & 5 & 100.00 \\
\hline \multirow[t]{3}{*}{ Defensa Central $(\mathrm{n}=5)$} & PL & $110.16 \pm 10.80$ & $107.04 \pm 13.31$ & $0.05 \pm 0.02$ & 3 & 60.00 \\
\hline & PM & $111.49 \pm 7.37$ & $112.27 \pm 8.28$ & $0.03 \pm 0.02$ & 2 & 40.00 \\
\hline & ANT & $105.00 \pm 15.30$ & $108.80 \pm 10.31$ & $0.05 \pm 0.06$ & 2 & 40.00 \\
\hline \multirow[t]{3}{*}{ Centrocampista ( $(\mathrm{n}=5)$} & PL & $114.14 \pm 17.14$ & $114.18 \pm 18.77$ & $0.10 \pm 0.09$ & 3 & 60.00 \\
\hline & PM & $112.70 \pm 12.59$ & $116.28 \pm 10.03$ & $0.04 \pm 0.04$ & 2 & 40.00 \\
\hline & ANT & $106.06 \pm 21.83$ & $105.92 \pm 18.69$ & $0.01 \pm 0.00$ & 0 & 00.00 \\
\hline \multirow[t]{3}{*}{ Extremo $(\mathrm{n}=2)$} & PL & $115.02 \pm 18.29$ & $111.74 \pm 22.50$ & $0.03 \pm 0.03$ & 1 & 50.00 \\
\hline & PM & $111.76 \pm 10.73$ & $111.12 \pm 9.82$ & $0.00 \pm 0.00$ & 3 & 00.00 \\
\hline & ANT & $110.94 \pm 16.77$ & $104.81 \pm 15.58$ & $0.05 \pm 0.08$ & 1 & 33.33 \\
\hline \multirow[t]{2}{*}{ Delantero $(\mathrm{n}=3$ ) } & PL & $117.02 \pm 13.51$ & $116.45 \pm 9.88$ & $0.06 \pm 0.02$ & 3 & 100.00 \\
\hline & PM & $119.20 \pm 15.12$ & $110.00 \pm 8.49$ & $0.12 \pm 0.09$ & 3 & 100.00 \\
\hline
\end{tabular}

Tabla 5

Alcance normalizado entre pierna dominante y no dominante y asimetrías entre extremidades: Diferencia entre pierna dominante y no dominante. $2^{\mathrm{a}}$ División B.

\begin{tabular}{|c|c|c|c|c|c|c|}
\hline \multirow{2}{*}{\multicolumn{2}{|c|}{ Posición en el campo }} & \multicolumn{2}{|c|}{ Alcance normalizado } & \multicolumn{3}{|c|}{ Déficit bilateral } \\
\hline & & Pierna & Pierna & Prom & Total $=$ & \\
\hline & ANT & $\begin{array}{l}\text { Dommante } \\
102.87 \pm 4.06\end{array}$ & $103.26 \pm 1.87$ & $0.01 \pm 0.01$ & $\begin{array}{l} \pm 4 \mathrm{~cm} \\
0\end{array}$ & $\begin{array}{l}10 \mathrm{da} \\
00.00\end{array}$ \\
\hline \multirow[t]{3}{*}{ Portero $(n=2)$} & PL & $118.24 \pm 3.03$ & $119.60 \pm 6.59$ & $0.02 \pm 0.02$ & 1 & 50.00 \\
\hline & PM & $118.89 \pm 18.89$ & $118.53 \pm 24.58$ & $0.04 \pm 0.01$ & 2 & 100.00 \\
\hline & & $4.41 \pm 10.89$ & $108.71 \pm 9.17$ & $0.03 \pm 0.02$ & 2 & 40.00 \\
\hline \multirow[t]{3}{*}{ Lateral $(n=5)$} & PL & $127.45 \pm 15$ & $133.45 \pm 13.25$ & $0.06 \pm$ & 2 & 40.00 \\
\hline & PM & $127.61 \pm 2$ & $136.18 \pm 1$ & $0.07 \pm$ & 3 & 60.00 \\
\hline & ANT & $104.41 \pm 11$ & $105.12 \pm 9$ & $0.02 \pm$ & 1 & 33.33 \\
\hline \multirow[t]{3}{*}{ Defensa Central $(\mathrm{n}=3)$} & PL & $118.12 \pm$ & $121.95 \pm$ & $0.03 \pm 0.02$ & 2 & 66.66 \\
\hline & PM & & & $0.05 \pm$ & 1 & 33.33 \\
\hline & ANT & & & $0.05 \pm$ & 3 & 75.00 \\
\hline \multirow[t]{3}{*}{ Centrocampista ( $\mathrm{n}=4)$} & PL & $003+$ & & $0.04 \pm$ & 3 & 75.00 \\
\hline & PM & & & $0.03 \pm$ & 1 & 25.00 \\
\hline & ANT & $109.81 \pm 1$ & $113.45 \pm$ & $0.03 \pm$ & 2 & 66.66 \\
\hline \multirow[t]{3}{*}{ Extremo $(n=3)$} & PL & $134.06 \pm 1$ & $136.14 \pm 8$ & $0.05 \pm$ & 1 & 33.33 \\
\hline & PM & & $139.39 \pm 1$ & $0.07 \pm c$ & 2 & \\
\hline & AN & $112.31 \pm 2.78$ & $112.61 \pm 9.71$ & $0.04 \pm 0.00$ & 2 & 100.00 \\
\hline De & & $125.22 \pm 19.42$ & $122.13 \pm 18.29$ & $0.02 \pm 0.01$ & 0 & \\
\hline
\end{tabular}

de $90^{\circ}$ para las medidas posterolateral y posteromedial y de $135^{\circ}$ de éstas respecto a la medida anterior (Gribble et al., 2016). Se colocó en primer lugar el $2^{\circ}$ dedo del pie derecho en el centro del eje de valoración, haciendo coincidir la dirección del talón con la medida anterior. El pie libre se colocó en paralelo al evaluado y las manos colocadas en la cintura, siendo considerada esta la posición de inicio. Se decidió colocar las manos en la cintura para facilitar el control por parte del observador, ya que, de esta manera si el sujeto perdía el equilibrio era fácilmente identificable al soltar las manos de la cintura.

Una vez establecida la posición de inicio, se solicitaba al participante que extendiese lo máximo posible la pierna libre en los tres planos de movimiento (ANT, PL y PM). La evaluación se realizó con ambas piernas (dominante y no dominante). Todos los sujetos estaban familiarizados con el test; realizándolo tres veces con cada pierna y seleccionando el valor máximo (Bahamonde et al., 2019). Atendiendo al estudio de (Langarica-Rocarfort, 2014), el intento no se consideró válido y se repitió si el jugador: soltaba las manos de la cadera, movía o levantaba el pie de apoyo en algún momento de la prueba, apoyaba el pie libre en el suelo, perdía el equilibrio desde que abandonaba la posición de inicio hasta que la volvía a retomar, y/o no era capaz de mantener la posición de inicio al menos un segundo después de retornar a la misma. En caso de errar en el intento, se recolocó al jugador en la posición de inicio y se volvía a repetir el intento. El intento se consideró válido, cuando el participante volvía al centro del eje de evaluación después de realizar los movimientos en los tres planos.

\section{Resultados}

Como se pueden observar en las tablas que se presentan a continuación, los resultados obtenidos por los 173 futbolistas de las cinco categorías muestran los datos según las posiciones que ocupaban estos en el campo. Los datos descriptivos del presente artículo se muestran en medias y sus desviaciones típicas para el YBT, con el fin de poder mostrar el alcance normalizado (pierna dominante, no dominante) y el déficit lateral (promedio $\pm \mathrm{DE}$; Total $\mathrm{d} » \pm 4 \mathrm{y} \%$ del total en $\mathrm{cm}$ ). Ver tabla 1 para más información.

A continuación, mostramos los resultados obtenidos por cada grupo en cada una de las categorías de competición.

En relación con los futbolistas de categoría cadete podemos observar en la tabla 2 toda la información obtenida para cada posición en el campo.

\begin{tabular}{|c|c|c|c|c|c|c|}
\hline & & \multicolumn{2}{|c|}{ Alcance normalizado } & \multicolumn{3}{|c|}{ Déficit bilateral } \\
\hline \multicolumn{2}{|l|}{ Posición en el campo } & Pierna & Pierna & Prom & Total $=$ & \% del \\
\hline \multirow{4}{*}{ Portero $(\mathrm{n}=3)$} & ANT & $62.26 \pm 10.60$ & $62.50 \pm 5.60$ & $0.03 \pm 0.03$ & 2 & 66.66 \\
\hline & PL & $115.41 \pm 4.59$ & $118.59 \pm 2.15$ & $0.01 \pm 0.01$ & 0 & 00.00 \\
\hline & PM & $114.01 \pm 5.08$ & $119.26 \pm 2.46$ & $0.03 \pm 0.03$ & 1 & 33.33 \\
\hline & ANT & $63.03 \pm 7.63$ & $64.41 \pm 4.71$ & $0.04 \pm 0.03$ & 2 & 50.00 \\
\hline \multirow[t]{3}{*}{ Lateral $(\mathrm{n}=4)$} & PL & $114.30 \pm 7.18$ & $116.98 \pm 6.60$ & $0.01 \pm 0.01$ & 0 & 00.00 \\
\hline & PM & $114.60 \pm 4.57$ & $114.02 \pm 4.73$ & $0.02 \pm 0.01$ & 1 & 25.00 \\
\hline & ANT & $61.15 \pm 9.33$ & $58.44 \pm 11.32$ & $0.04 \pm 0.03$ & 1 & 33.33 \\
\hline \multirow[t]{3}{*}{ Defensa Central $(n=3)$} & PL & $114.80 \pm 3.89$ & $121.17 \pm 2.64$ & $0.07 \pm 0.04$ & 2 & 66.66 \\
\hline & PM & $113.90 \pm 8.08$ & $115.71 \pm 1.80$ & $0.03 \pm 0.04$ & 2 & 33.33 \\
\hline & ANT & $59.23 \pm 6.45$ & $59.87 \pm 6.85$ & $0.04 \pm 0.02$ & 3 & 50.00 \\
\hline \multirow[t]{3}{*}{ Centrocampista $(\mathrm{n}=6)$} & PL & $111.04 \pm 10.84$ & $111.04 \pm 10.84$ & $0.06 \pm 0.02$ & 5 & 83.33 \\
\hline & PM & $107.92 \pm 10.06$ & $105.35 \pm 9.23$ & $0.06 \pm 0.04$ & 2 & 33.33 \\
\hline & ANT & $62.12 \pm 3.62$ & $61.39 \pm 4.74$ & $0.02 \pm 0.01$ & 0 & 00.00 \\
\hline \multirow[t]{3}{*}{ Extremo $(n=4)$} & PL & $118.63 \pm 4.10$ & $115.95 \pm 5.18$ & $0.01 \pm 0.01$ & 1 & 25.00 \\
\hline & PM & $120.89 \pm 2.48$ & $118.16 \pm 4.40$ & $0.01 \pm 0.01$ & 1 & 25.00 \\
\hline & ANT & $64.33 \pm 8.54$ & $65.72 \pm 7.05$ & $0.01 \pm 0.01$ & 0 & 00.00 \\
\hline \multirow[t]{2}{*}{ Delantero $(\mathrm{n}=3)$} & PL & $117.73 \pm 12.59$ & $119.94 \pm 11.57$ & $0.03 \pm 0.01$ & 2 & 66.66 \\
\hline & PM & $113.14 \pm 8.54$ & $118.41 \pm 8.74$ & $0.04 \pm 0.01$ & 3 & 100.00 \\
\hline
\end{tabular}


En la tabla 3 se muestran los resultados obtenidos por los futbolistas de categoría juvenil. Observar tabla 3 para más información.

A continuación, se muestran los resultados obtenidos

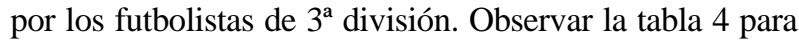
más información.

En la tabla 5 se muestran los resultados obtenidos por los futbolistas de $2^{\text {a }}$ División B para cada una de las categorías. Observar tabla 5 para más información.

Por último, en la tabla 6 se muestran los resultados obtenidos por futbolistas profesionales de $1^{\mathrm{a}}$ DivisiónObservar tabla 6 para más información.

\section{Discusión}

El propósito del presente estudio radica en mostrar los rangos de normalidad para jugadores de fútbol en diferentes categorías, atendiendo a la estabilidad postural y el rango de movimiento evaluado a través del YBT. Para alcanzar el anterior objetivo, se han analizado a jugadores de fútbol de diferente edad y categorías (Cadete, Juvenil, $3^{\text {a }}$ División, $2^{\text {a }}$ división B y $1^{\text {a }}$ División) y diferenciando la posición específica. La selección del YBT como método de medición del rango de movimiento en las piernas y su evaluación para control postural dinámico, tiene una contrastada evidencia para relacionar los resultados obtenidos con una mayor o menor probabilidad de lesiones en las extremidades inferiores (Butler et al., 2012; Gribble et al., 2016; Plisky et al., 2006).

Hasta donde alcanza nuestro conocimiento, no conocemos ningún estudio descriptivo y longitudinal que establezca rangos de normalidad del YBT para jugadores de fútbol, de ahí nuestro interés en este importante tópico de investigación. De hecho, nuestra principal aportación resulta en la generación de un marco de referencia para los profesionales del fútbol, teorizando sobre los rangos de normalidad por cada categoría y puesto. Este conocimiento viene justificado, ya que, se estima que entre el $68-88 \%$ de totalidad las lesiones producidas en el fútbol se registran en el tren inferior tanto en jugadores profesionales como de categorías inferiores (Ekstrand, Hägglund, \& Waldén, 2011; Junge, Cheung, Edwards, \& Dvorak, 2004). Además, hay evidencias propuestas en la literatura que inciden en la importancia de conocer y normalizar unos valores de referencia en el YBT para así identificar a aquellos que se desvían de los promedios (Stiffler et al., 2015; 2017).

Otra importante aportación, es que, se consideran los resultados presentandos en esta investigación como un punto de referencia para evaluar el comportamiento del equilibrio dinámico y el aumento de posibles dismetrías en diferentes categorías y puestos específico de futbolistas. En relación a esta idea, se ha demostrado que el equilibrio dinámico mejora a medida que aumenta el nivel competitivo, existiendo una tendencia a la disminución de las asimetrías a medida que aumenta la edad de entrenamiento (Butler et al., 2012). Los datos extraídos de nuestra investigación coinciden con los presentados por Butler et al., (2012), ya que, como se observa en las tablas 2-6, el comportamiento del déficit bilateral va disminuyendo a medida que aumenta la categoría. En adicción a esta aportación, parece existir evidencia que indica una suavización de las asimetrías en los jugadores de fútbol con una mayor práctica de entrenamiento más larga, reduciendo potencialmente el riesgo de lesión (Fousekis, Tsepis, \& Vagenas, 2012). Por lo tanto, la relevancia de los datos descritos se torna realmente importante ya que es fundamental conocer el comportamiento de estas dismetrías tan variables a lo largo de diferentes etapas. Estos desajustes encontrados en edades más tempranas, podrían ser debido a que los 14-15 años (cadete) es una edad en continuo desarrollo corporal que no terminar de estabilizarse aproximadamente hasta los 21 años, por lo qu,e los jugadores se enfrenan un desajuste corporal que es transcendental controlar para evitar posibles lesiones (Soarez, Fragoso, Massuça, \& Barrigas, 2012). Al mismo tiempo, hay que añadir a esta idea los resultados encontrados por Hall, Foss, Hewett, \& Myer, (2015), donde se destaca que los jugadores que participan en una solo deporte tienen un riego 1,5 veces mayor probabilidad de sufrir lesiones en el tren inferior que los jugadores que hace una práctica multideportiva.

Las investigaciones realizadas por Butler et al., (2012), evidenciaron un mayor equilibro dinámico en las tres medidas (ANT, PL y PM), en jugadores profesionales con respecto a los amateurs. Coincidiendo con esta idea, nuestro estudio ha constado que en las categorías seniors no parece existir ningún patrón de comportamiento de asimetrías para ninguna de las tres medidas, estableciéndose un comportamiento más estable en el equilibrio dinámico. Estos datos podrían estar explicado por el conjunto de mejores actitudes en el rango de movimiento, la coordinación, la fuerza y propiocepción de los jugadores de fútbol más experimentados (Butler et al., 2012). El único matiz detectado, es que, en categorías seniors y en el caso de los centrocampistas, siempre se destaca un mayor desajuste en PL destacado por encima del resto de medidas. Bahamonde et al., (2019), realizaron una investigación sobre como un programa de intervención de actividades extraescolares mejoraba el equilibrio dinámico evaluado a través del Y-Balance test en jóvenes futbolistas. Los resultados obtenidos en estos test no difieren en exceso con los presentando en nuestra investigación. Ahora bien, hay que matizar que los datos presentado por Bahamonde et al., (2019), son generales y encuentran desacuerdo con otras investigaciones como las de los Linek et al., (2017). Como se observa, nuestro estudio tiene la importancia de establecer referentes teóricos por posiciones y niveles de competición, por lo que podrían servir para explicar en parte, las posibles diferencias encontradas entre diseños de estudio similares

Sin embargo, para el resto de categorías hemos detectado una serie de patrones. Por ejemplo, en el caso de los delanteros y para todas las categorías siempre se destaca un mayor déficit postora medial (PM). Otro aspecto destacable existe en la categoría cadete donde se destacan a los centrocampistas como la posición que más déficit ofrece en todas las medidas bilaterales, situación que también sucede en la categoría juvenil para dos de las tres evaluaciones (PL y PM). Robinson \& Gribble (2008), subrayan la importancia de la flexión de la rodilla de la pierna de apoyo como el predictor más fuerte para determinar los resultados de medida en las direcciones posterolateral y posteromedial.

Que existan diferencias bilaterales en determinadas posiciones, puede quedar explicado por las propias demandas 
físicas intrínsecas al fútbol, ya que, la realización del YBT requiere de una mayor movilidad de la cadera, conllevando una mayor movilidad de la musculatura de la cadena posterior y el control excéntrico durante la flexión de cadera (Robinson \& Gribble, 2008). Además, hay que añadir la importancia de la fuerza isométrica necesaria en las extremidades inferiores, aún por desarrollar en categorías inferiores, ya que, se ha evidenciado que esta tiene una relación significativa positiva con el equilibrio dinámico cuando se evaluara a través del YBT (Chtara et al., 2018).

\section{Conclusiones}

La presenten investigación se ha centrado en la generación de un marco conceptual a la par que una aproximación teórica que sirva de marco de referencia para futuras investigaciones que tengan como objetivo medir los déficits bilaterales en futbolistas con YBT. Por lo tanto, el objetivo de este estudio ha sido establecer los rangos de normalidad en el test Y-Balance en jugadores de fútbol, atendiendo a la categoría (cadete, juvenil, $3^{\text {a }}$ división, $2^{\text {a }}$ división B y $1^{\text {a }}$ división) y puestos específicos.

Además de la generación de este este marco de referencia, podemos establecer como principal conclusión la existencia de una mejora de los resultados en YBT a medida que aumenta la categoría para todas las medidas evaluadas. Otra conclusión secundaria es la detección de una serie de patrones de déficit en determinadas posiciones, como sucede en los casos de los delanteros de todas las categorías con la medida posteromedial (PM). En esta descripción también se observa como tanto en las categorías cadetes como juveniles los centrocampistas es la posición que más déficits aparecen.

Una vez generado este marco de referencia, consideramos la realización de futuras investigaciones donde comparar las posibles diferencias significativas entre jugadores de la misma posición, pero diferentes categorías de rendimiento (amateur, semiprofesional y profesionales) para así conocer hasta qué punto la influencia del puesto específico puede generar ciertas asimetrías. Al mismo tiempo, dadas las pocas investigaciones realizadas con jugadores profesionales de fútbol es importante conocer bien el comportamiento de los resultados obtenidos con YBT para así conocer con mayor precisión como profesionalización influye en las asimetrías y así generar programas preventivos más eficientes.

\section{Referencias}

Ageberg, E., Roberts, D., Holmström, E., \& Fridén, T. (2005). Balance in single-limb stance in patients with anterior cruciate ligament injury: relation to knee laxity, proprioception, muscle strength, and subjective function. American Journal of Sports Medicine, 33(10), 1527-1537.

Atkins, S. J., Bentley, I., Hurst, H. T., Sinclair, J. K., \& Hesketh, C. (2016). The presence of bilateral imbalance of the lower limbs in elite youth soccer players of different ages. Journal of Strength \& Conditioning Research, 30(4), 1007-1013.

Bahamonde, C., Carmona, C., Albornoz, J., Hernández-Garcia, R., \& Torres-Luque, G. (2019). Efecto de un programa de actividades deportivas extraescolares en jóvenes chilenos. RETOS: nuevas tendencias en educación física, deporte y recreación, (35), 261-266.

Bressel, E., Yonker, J. C., Kras, J., \& Heath, E. M. (2007). Comparison of Static and Synamic Balance in Female. Journal of Athletic Training, 42(1), 42-46.

Butler, R. J., Queen, R. M., Beckman, B., Kiesel, K. B., \& Plisky, P. J. (2013). Comparison of dynamic balance in adolescent male soccer players from Rwanda and the United States. International Journal of Sports Physical Therapy, 8(6), 749.

Butler, R. J., Southers, C., Gorman, P. P., Kiesel, K. B., \& Plisky, P. J. (2012). Differences in soccer players' dynamic balance across levels of competition. Journal of Athletic Training, 47(6), 616-620.

Chtara, M., Rouissi, M., Bragazzi, N. L., Owen, A. L., Haddad, M., \& Chamari, K. (2018). Dynamic balance ability in young elite soccer players: implication of isometric strength. Journal of Sports Medicine and Physical Fitness, 58(4), 414-420.

Ekstrand, J., Hägglund, M., \& Waldén, M. (2011). Injury incidence and injury patterns in professional football: The UEFA injury study. British Journal of Sports Medicine, 45(7), 553-558.

Fousekis, K., Tsepis, E., \& Vagenas, G. (2012). Intrinsic risk factors of noncontact ankle sprains in soccer: A prospective study on 100 professional players. American Journal of Sports Medicine, 40(8), 1842-1850.

Gonell, A. C., Romero, J. A. P., \& Soler, L. M. (2015). Relationship between the $\mathrm{Y}$ balance test scores and soft tissue injury incidence in a soccer team. International Journal of Sports Physical Therapy, 10(7), 955.

González, G., Oyarzo, C., Fischer, M., De la Fuente, M. J., Diaz, V., \& Berral, F. J. (2011). Specific training of the postural balance in the young soccer players. Revista Internacional de Medicina y Ciencias de La Actividad Física y El Deporte, 41, 95-114.

Gribble, P. A., \& Hertel, J. (2003). Considerations for normalizing measures of the Star Excursion Balance Test. Measurement in Physical Education and Exercise Science, 7(2), 89-100.

Gribble, P.A., Hertel, J., Facsm, À., \& Plisky, P. (2007). Chapter 7 Applications. North-Holland Mathematics Studies, 207(3), 131-152.

Gribble, P. A., Hertel, J., \& Plisky, P. (2012). Using the Star Excursion Balance Test to assess dynamic postural-control deficits and outcomes in lower extremity injury: a literature and systematic review. Journal of Athletic Training, 47(3), 339-357.

Gribble, P.A., Terada, M., Beard, M. Q., Kosik, K. B., Lepley, A. S., McCann, R. S., ... Thomas, A. C. (2016). Prediction of Lateral Ankle Sprains in Football Players Based on Clinical Tests and Body Mass Index. American Journal of Sports Medicine, 44(2), 460-467.

Gribble, P. A., Tucker, W. S., \& White, P. A. (2007). Time-ofday influences on static and dynamic postural control. Journal of Athletic Training, 42(1), 35.

Hall, R., Foss, K. B., Hewett, T. E., \& Myer, G. D. (2015). Sport specialization's association with an increased risk of developing anterior knee pain in adolescent female 
athletes. Journal of Sport Rehabilitation, 24(1), 31-35.

Hertel, J., Braham, R. A., Hale, S. A., \& Olmsted-Kramer, L. C. (2006). Simplifying the Star Excursion Balance Test: Analyses of Subjects With and Without Chronic Ankle Instability. Journal of Orthopaedic \& Sports Physical Therapy, 36(3), 131-137.

Hewit, J. K., Cronin, J. B., \& Hume, P.A. (2012). Asymmetry in multi-directional jumping tasks. Physical Therapy in Sport, 13(4), 238-242.

Junge, A., Cheung, K., Edwards, T., \& Dvorak, J. (2004). Injuries in youth amateur soccer and rugby players Comparison of incidence and characteristics. British Journal of Sports Medicine, 38(2), 168-172.

Keith, T. R., Condon, T. A., Phillips, A., McKeon, P. O., \& King, D. L. (2016). Postural control strategies are dependent on reach direction in the star excursion balance test. International Journal of Athletic Therapy and Training, 21(6), 33-39.

Mancera-Soto, E., Hernández-Álvarez, É., Hernández-Salinas, F., Prieto-Mondragon, L., \& Quiroga-Díaz, L. (2013). Effect of physical training based on the developmental sequence of postural balance in football players: randomized controlled trial. Revista de la Facultad de Medicina, 61(4), 339-347.

Mcguine, T. ., Greene, J. J., Best, T., \& Leverson, G. (2000). Balance as a predictor of ankle injuries in high school basketball players. ClinicalJournal of Sport Medicine, 10(4), 239-244.

Meardon, S., Klusendorf, A., \& Kernozek, T. (2016). Influence of injury on dynamic postural control in runners. International Journal of Sports Physical Therapy, 11(3), 366.

Langarica-Rocafort, A. (2014). Fiabilidad de diferentes pruebas que se utilizan para evaluar el riesgo de lesión del miembro inferior en mujeres deportistas. (Doctoral dissertation, Servicio Editorial de la Universidad del País Vasco/Euskal Herriko Unibertsitatearen Argitalpen Zerbitzua).

Leirós-Rodríguez, R., Arce-Fariña, M., \& García-Soidán, J.L. (2015). Estudio piloto para la valoración del equilibrio en mujeres adultas con un elemento cinemático. RETOS. Nuevas Tendencias en Educación Física, Deporte y Recreación, (28), 90-93.

Lee, J., Lin, A., \& Huang, C. (2006). Impaired proprioception and poor static postural control in subjects with functional instability. Journal of Exercise Science and Fitness, 4(2), 117-125.

Linek, P., Sikora, D., Wolny, T., \& Saulicz, E. (2017). Reliability and number of trials of Y Balance Test in adolescent athletes. Musculoskeletal Science and Practice, 31: 7275.

Plisky, P. J., Gorman, P. P., Butler, R. J., Kiesel, K. B., Underwood, F. B., \& Elkins, B. (2009). The reliability of an instrumented device for measuring components of the star excursion balance test. North American Journal of Sports Physical Therapy, 4(2), 92.

Plisky, P. J., Rauh, M. J., Kaminski, T. W., \& Underwood, F. B. (2006). Star Excursion Balance Test as a Predictor of Lower Extremity Injury in High School Basketball Players. Journal of Orthopaedic \& Sports Physical Therapy,
36(12), 911-919.

Robinson, R., \& Gribble, P. (2008). Kinematic Predictors of Performance on the Star Excursion Balance Test. Journal of Sport Rehabilitation, 17(4), 347-357.

Sclafani, M. P., \& Davis, C. C. (2016). Return to play progression for rugby following injury to the lower extremity: a clinical commentary and review of the literature. International Journal of Sports Physical Therapy, 11(2), 302.

Soarez, H., Fragoso, I., Massuça, L., \& Barrigas, C. (2012). Impacto de la maduración y de los puestos específicos en la condición física en jóvenes futbolistas. Apunts. Medicina de l'Esport, 47(174), 73-81.

Stiffler, A. M. R., Bell, D. R., Sanfilippo, J. L., Hetzel, S. J., Pickett, K. A., \& Heiderscheit, B. C. (2015). Star Excursion Balance Test Performance Varies by Sport in Healthy Division I Collegiate Athletes. Journal of Orthopaedic \& Sports Physical Therapy, 45 (10), 772-780.

Stiffler, M. R., Bell, D. R., Sanfilippo, J. L., Hetzel, S. J., Pickett, K.A., \& Heiderscheit, B. C. (2017). Star Excursion Balance Test Anterior Asymmetry is Associated with Injury Status in Division I Collegiate Athletes. Sports Physical Therapy, 47(5), 339-346.

Triana, F. C., \& Espitia, J. E. B. (2019). Confiabilidad de los test que miden las capacidades coordinativas en deportes acíclicos. Revista digital: Actividad Física y Deporte, 5(1), 51-66.

Troule, S., \& Casamichanana, D. (2016). Aplicación de pruebas funcionales para la detección de asimetrías en jugadores de fútbol. Journal of Sport and Health Research, 8(1), 53-64.

Vanmeerhaeghe, A. F., Rodríguez, D. R., Tutusaus, L. C., Calafat, C. B., Riera, M. L., \& Vidal, A. M. (2009). Diferencias en la estabilidad postural estática y dinámica según sexo y pierna dominante. Apunts. Medicina de l'Esport, 44(162), 74-81.

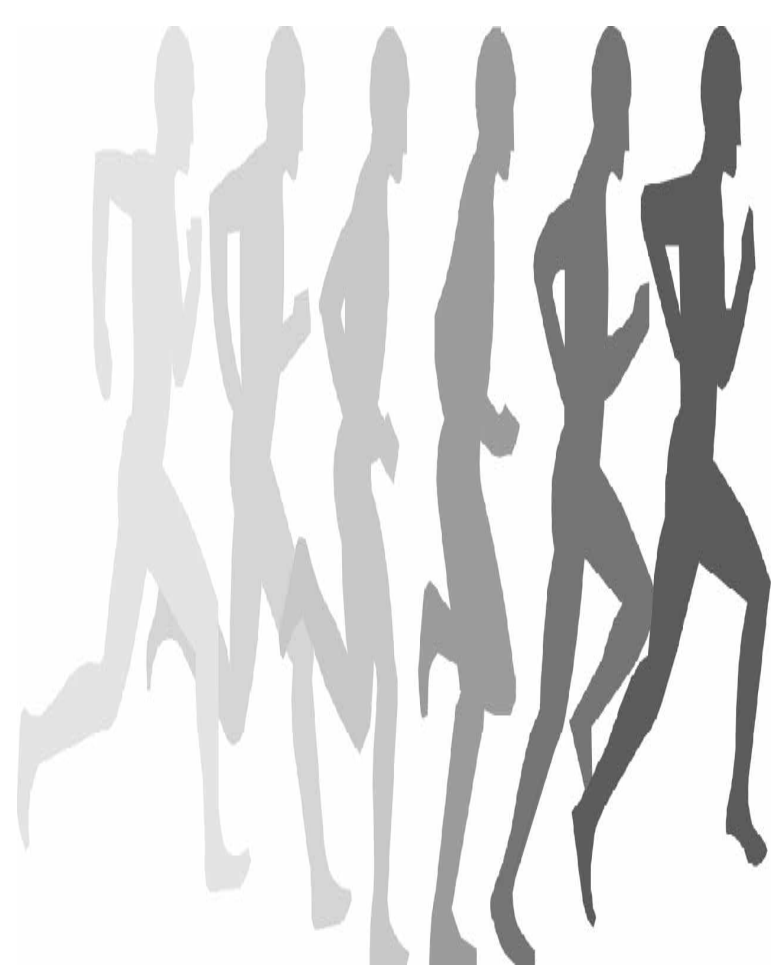

